RESEARCH NOTE

\section{Effects of Chronic Chagasic Infection on the Number and Size of Cardiac Neurons of the Wild Rodent Calomys callosus}

\section{Romeu R De-Souza, Denise C Vasconcelos, Laura BM Maifrino*, Edson A Liberti/ ${ }^{+}$}

\begin{abstract}
Laboratório de Estudos do Sistema Nervoso Autônomo, Departamento de Anatomia, Instituto de Ciências Biomédicas, Universidade de São Paulo, Av. Prof. Lineu Prestes 2415, 05508-900 São Paulo, SP, Brasil *Instituto Dante Pazzanese de Cardiologia, Av.

Dante Pazzanese 500, 04012-180 São Paulo, SP, Brasil
\end{abstract}

Key words: cardiac neurons - experimental Chagas disease - neuronal counting - Calomys callosus

Calomys callosus is a wild rodent which was found harbouring Trypanosoma cruzi (RD Ribeiro 1973 Clin Exp Immunol 3: 429-537). This animal characteristically exhibit low mortality when infected with $T$. cruzi, in contrast with other species (MM Borges et al. 1992 Mem Inst Oswaldo Cruz 87: 493-502). The experimental model of Chagas disease has mostly been the mouse and experimental infection of wild rodents is little explored (Borges et al. loc. cit.). To our knowledge, no papers are available concerning the cardiac neurons of $C$. callosus in Chagas disease. The objective of this work was to analyze the effects of chronic chagasic infection on the number and size of cardiac neurons of this animal species.

Seven specimens of $C$. callosus males, aged one month, weighing 30-40 g, were inoculated with $1 \times 10^{5}$ of the Y strain of $T$. cruzi. Other seven $C$. callosus of the same strain and weight were used as controls. Control and T. cruzi infected animals were killed 60 days after inoculation. All the ani-

\footnotetext{
${ }^{+}$Corresponding author. Fax: + 55-11-813.0845. E- mail: ealibert@biomed.icb.usp.br

Received 19 November 1998

Accepted 17 May 1999
}

mals were sacrificed with an overdose of ether. The thorax and pericardium were opened, and the heart removed. Under the stereomicroscope, the atria were separated from the ventricles and dissected in order to remove excess of fat and connective tissue from their external surface. The aorta, pulmonary trunk and interatrial septum were removed. The neurons were stained by a histochemical technique to detect DPNH-reductase activity (G Gabella 1969 Experientia 25: 218-19). The atria were then fixed in $10 \%$ neutral formalin and wholemount in glycerin jelly for microscopic examination. Neuron counts were made directly on the whole-mount preparations under the microscope (magnification of $125 \mathrm{X}$ ); data were tabulated and averages and standard deviations calculated. For estimation of neuron size, the profiles of 50 randomly chosen nerve cell perikarya for each animal were outlined on drawing paper using a camera lucida attached to a microscope (1200 X). The areas of these cell bodies were estimated using a planimeter OTT-30. The ranges of cardiac neuron size groups (control and chagasic) were distributed in histograms of the frequencies, which were compared by the Chi-square test.

In the whole-mount preparations of $C$. callosus atria from the control group, numerous ganglion neurons were found. The neurons occurred commonly in clusters that included up to 50 neurons (Fig. 1 A, B). The cardiac ganglia were found exclusively on the external surface of atrial muscle, in the subepicardial connective tissue. In the chronic chagasic animals several ganglia showed the neurons less densely packed and the shape of the neurons was altered. Other ganglia contained destroyed neurons (Fig. 1 C-F). No inflammatory infiltrate has been observed because the staining technique does not allow this determination.

The average number of cardiac neurons from seven atria of chagasic $C$. callosus was $446 \pm 151$ (mean \pm standard deviation) while animals from control group showed $659 \pm 120$ neurons. The difference in the number of neurons between the two groups was significant $(\mathrm{p}<0.05)$. The size of neurons was assessed (as cell profile area) in the wholemount preparations used for cell counts. Sizes ranged from about $100 \mu \mathrm{m}^{2}$ to about $900 \mu \mathrm{m}^{2}$ in all the animals, infected and non infected (Fig. 2). The mean was $370 \mu \mathrm{m}^{2}$ in controls while $426 \mu \mathrm{m}^{2}$ in chagasic animals. The difference in size between control and chagasic C. callosus was statistically significant $(\mathrm{p}<0.05)$.

The results of the present work are based on neuronal counts performed in whole-mount preparations of the atrial wall. The histochemical method used was adequate to stain the cardiac neurons, confering to the membranes specificity and uni- 

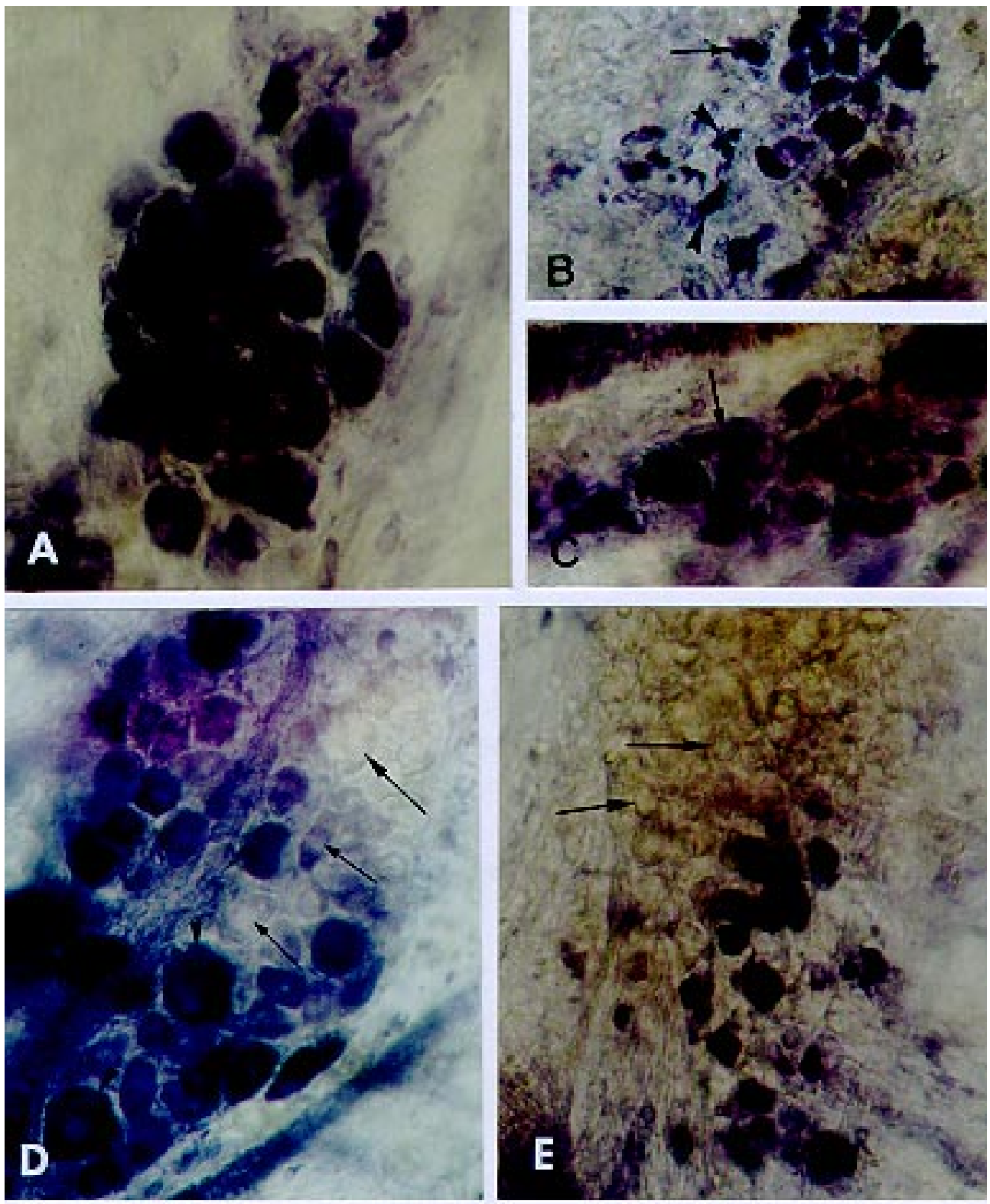

Fig. 1 A-E: histochemically stained cardiac ganglia. A: ganglion from a control Calomys callosus showing a densely packed cluster of neurons. Ganglion neurons are mainly round in shape. X 320; B, C: chagasic C. callosus. Ganglion neurons are irregular in shape (arrows) and some of them seem to be degenerated (arrowheads). X 256; D, E: ganglia from chagasic C. callosus, with most of the neurons poorly stained or not stained (arrows). Two large neurons can also be seen (arrowheads). X 256.

formity in staining and it was possible to count the neurons, obtaining values more reliable than those achieved with histological sections. In addition, completely destroyed neurons and alterations in the shape of several cardiac neurons produced by the chronic $T$. cruzi infection were observed.

The average number of cardiac neurons of chagasic $C$. callosus $(446 \pm 151)$ was smaller than the average number of the control group $(659 \pm$
120) and the difference was statistically significant $(\mathrm{p}<0.05)$. The change in total number of neurons is, therefore, interpreted as indicating that about $24 \%$ of the neuronal population disappear from the ganglia of infected animals. The loss of cardiac neurons is due to nerve cell destruction. Studies carried out in the heart of other chagasic animal species (F Köberle 1959 Rev Med Córdoba 47: 105-133, FG Alcântara 1970 Rev Goiana Med 16: 

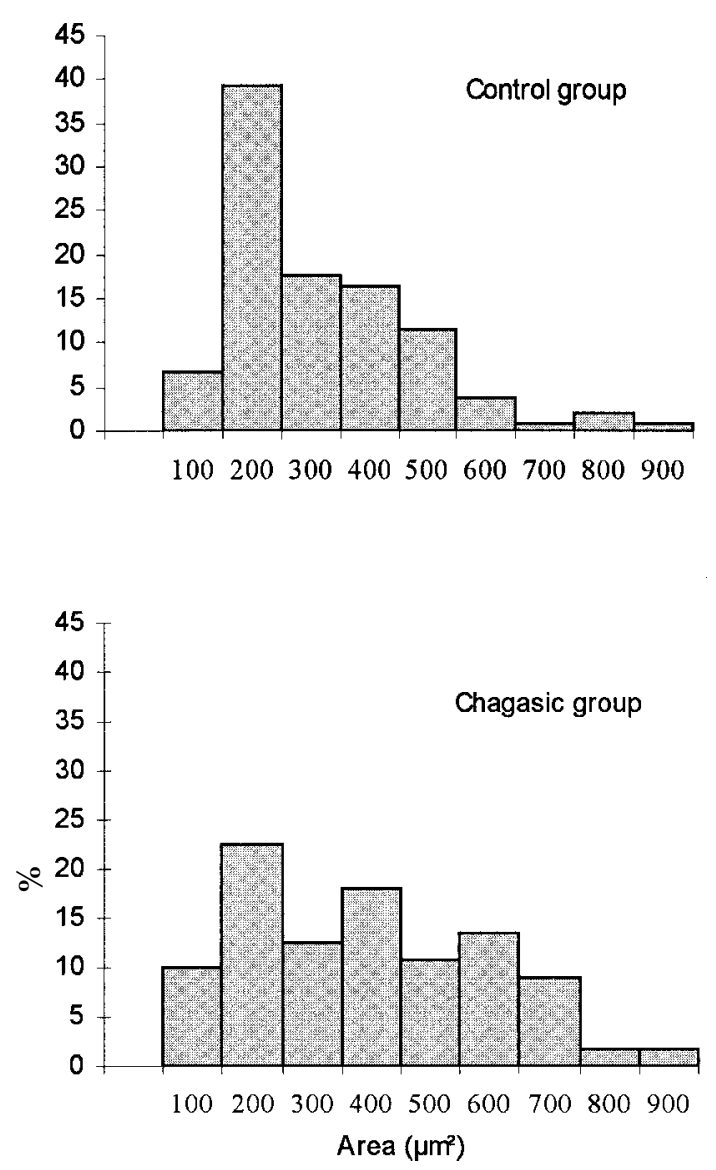

Fig. 2: effect of chronic chagasic infection on atrial neuron cell size. Data are reported as histograms of neuronal cell profile area for 350 neurons measured in heart tissue from seven Calomys callosus for each group.

159-177, WL Tafuri 1970 Am J Trop Med Hyg 19: 405-417, RB Ribeiro dos Santos \& L Hudson 1981 Clin Exp Immunol 44: 349-354, DF Davila et al. 1995 Rev Inst Med Trop de São Paulo 37: 155-59) and in human hearts (JSM Oliveira 1985 Am Heart $J$ 100: 1092-1098) also showed reduction in the number of cardiac neurons. The denervation detected in the cardiac ganglia of C. callosus is lower than that observed in other species, except for the rat, in which no significant reduction in the num- ber of neurons in chagasic animals was observed (E Chapadeiro et al. 1991 Rev Inst Med Trop São Paulo 33: 337-341).

In terms of cell size the cardiac neurons of $C$. callosus do not form an uniform population. They range in the area of their profile from about 100 $\mu \mathrm{m}^{2}$ to about $900 \mu \mathrm{m}^{2}$. The comparison between the control and chagasic animals showed that the spread of neuronal sizes is the same in both groups. However, while in control C. callosus about 70\% of the neurons measure between 300 and $500 \mu \mathrm{m}^{2}$ (with a peak at $300 \mu \mathrm{m}^{2}$ ) in chagasic animals the same proportion of neurons spreads between 300 and $700 \mu \mathrm{m}^{2}$ without a distinct peak. The histogram from the chagasic group shows a shift to the right, and the mean of nerve cell body areas in the chagasic animals was greater than that of the control group. These figures indicate an enlargement of neuronal cell bodies during the infection. The causes of this process are unknown but it is possible that some compensatory growth occurs in the remaining neurons as an attempt to maintain the density of the innervation of the cardiac tissues.

It has been suggested that lesions in cardiac neurons could be related to the depression of the baroreflex autonomic control of cardiac rhythm observed in chronic chagasic patients (DS Amorim et al. 1968 Circulation 38: 289-294, DJ Iosa et al. 1980 J Auton Nerv Syst 2: 87-92, LF Junqueira Jr 1990 Braz J Med Biol Res 23: 1091-1102). However, in an experiment with chagasic rats (Chapadeiro et al. loc. cit.), although the heart parasympathetic dysfunction characterized by reduction in the index of bradycardia baroreflex responsiveness was shown, the microscopic examination of the atrial ganglia, showed no statistically significant reduction in the number of neurons. These data suggest that the parasympathetic dysfunction may not depend exclusively on the lesions of the heart neurons (Chapadeiro et al. loc. cit.) and, therefore, other experiments may be necessary to elucidate this question.

The chronic T. cruzi infection in C. callosus can be a useful model for the study of the mechanisms of cardiopathies produced by lesions in cardiac neurons. 
